International Journal of Bifurcation and Chaos, Vol. 22, No. 7 (2012) 1250173 (13 pages)

(c) World Scientific Publishing Company

DOI: $10.1142 / \mathrm{S} 0218127412501738$

\title{
BIFURCATIONS IN A STAR-LIKE NETWORK OF STUART-LANDAU OSCILLATORS
}

\author{
MATTIA FRASCA* ${ }^{*}$ ANDRÉ BERGNER ${ }^{\dagger}$, JÜRGEN KURTHS $^{\ddagger}$ \\ and LUIGI FORTUNA* \\ *Laboratorio sui Sistemi Complessi, Scuola Superiore di Catania, \\ Dipartimento di Ingegneria Elettrica Elettronica e Informatica, \\ Facoltà di Ingegneria, Università degli Studi di Catania, Italy \\ †Institute of Physics, University of Potsdam, \\ 14476 Potsdam, Germany \\ ${ }^{\ddagger}$ Potsdam Institute for Climate Impact Research (PIK), \\ 14473 Potsdam, Germany

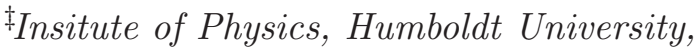 \\ Berlin, Germany \\ $¥$ Institute for Complex Systems and Mathematical Biology, \\ University of Aberdeen, Aberdeen AB243UE, UK
}

Received November 16, 2011; Revised March 8, 2012

\begin{abstract}
In this paper, we analytically study a star motif of Stuart-Landau oscillators, derive the bifurcation diagram and discuss the different forms of synchronization arising in such a system. Despite the parameter mismatch between the central node and the peripheral ones, an analytical approach independent of the number of units in the system has been proposed. The approach allows to calculate the separatrices between the regions with distinct dynamical behavior and to determine the nature of the different transitions to synchronization appearing in the system. The theoretical analysis is supported by numerical results.
\end{abstract}

Keywords: Complex networks; synchronization; bifurcations.

\section{Introduction}

Synchronization, i.e. the process wherein two or more dynamical systems (either identical or nonidentical), coupled through suitable configurations or driven by a common forcing signal, can coordinate a particular dynamical property, is a complex emerging phenomenon appearing in many complex systems: brain [Varela et al., 2001], coupled circuits [Arena et al., 2006, 2008; Fortuna \& Frasca, 2007; Buscarino et al., 2009], coupled mechanical systems [Pantaleone, 2002], lasers [Meucci et al., 2006], Josephson junctions [Wiesenfeld et al., 1996], social systems [Neda et al., 2000], climate
[Mokhov et al., 2011], cardiovascular systems [Van Leeuwen et al., 2009] and many other examples [Pikovsky et al., 2003; Strogatz, 2003; Boccaletti et al., 2002]. The phenomenon has been studied both in systems of coupled periodic oscillators [Strogatz, 2000] and in coupled chaotic systems [Boccaletti et al., 2002], and several types of synchronization have been identified [Boccaletti et al., 2002]: complete, phase, lag, generalized, intermittent, imperfect or almost synchronization.

The first works on synchronization of chaotic systems appeared in the 80's [Fujisaka \& Yamada, 1983; Afraimovich et al., 1986]. Later on, in 90's, 
after synchronization in two coupled circuits was demonstrated [Pecora \& Carroll, 1990], the focus was mainly on synchronization of two dynamical units and related techniques for the design of suitable coupling configurations. Then, progressively, the focus moved on to synchronization of networks of dynamical units: from regular structures [Wu \& Chua, 1995] to complex networks [Boccaletti et al., 2006]. In this latter case, a fundamental result is represented by the master stability function [Pecora \& Carroll, 1998], an approach that allows to derive the analytical conditions under which a group of identical coupled dynamical systems can show a stable synchronous behavior.

However, since most real systems are not formed by identical units, there is a great interest to investigate both systems constituted by nearly identical dynamical units and systems formed by nodes ruled by nonidentical dynamical equations. The first case has been dealt with for instance in [Sun et al., 2009] where the master stability function approach has been then generalized to account also for the case of nearly identical units. The second case is currently the subject of intense research [Chen \& Lu, 2008; Amriktar \& Rangarajan, 2009; Zhao et al., 2011].

Another issue which is attracting growing interest is the role played by motifs in synchronization of complex networks. Motifs are specific patterns of interconnections occurring in a network (either direct or indirect) at a rate significantly higher than in randomized versions of the network [Lodato et al., 2007]. Therefore, network motifs can be viewed as major constituents of larger networks of oscillators, and the investigation of their synchronization properties can provide significant insights to the understanding of synchronization in larger networks. The synchronization properties of motifs of three- and four-nodes have been studied in [Lodato et al., 2007], while unidirectional rings, bidirectional rings and open chains of Kuramoto oscillators have been investigated in [D'Huys et al., 2008].

In this work, we consider that the node dynamics is represented by a Stuart-Landau oscillator. Topologies of networks of coupled Stuart-Landau oscillators such as all-to-all networks [Matthews \& Strogatz, 1990; Ermentrout, 1990; Hakim \& Rappel, 1992; Nakagawa \& Kuramoto, 1993] and arrays of Stuart-Landau oscillators [Mirollo \& Strogatz, 1990] have been studied in several works. Networks of Stuart-Landau oscillators are also often used as models of oscillator neural networks [Aoyagi, 1995; Kawaguchi, 2000; Hoppensteadt \& Izhikevich, 2001; Burwick, 2006; Uchiyama, 2012]. In this work, we investigate a specific motif, namely a star network formed by nonidentical Stuart-Landau units. In particular, the $h u b$ oscillator and the leaves have strongly different parameters. This motif has recently attracted a lot of attention, since two very interesting synchronization phenomena have been observed in such topologies: star networks, and more in general scale-free networks, of Kuramoto oscillators have been found to exhibit explosive synchronization, i.e. a transition from the nonsynchronized to synchronized regime occurring through a second-order transition [Gómez-Gardeñes et al., 2011]; star networks of Stuart-Landau oscillators have been instead found to exhibit remote synchronization, a regime in which the leaves are synchronized with each other, but not with the hub [Bergner et al., 2012]. In this work we focus on the star network formed by Stuart-Landau oscillators and analytically derive the bifurcation diagram of this system. The behavior of the system is studied with respect to two parameters: the coupling strength and the parameter mismatch (which in our case represents the mismatch between the natural frequency of the hub and that of the leaves). For small enough frequency mismatches synchronization in the system is observed. More precisely, phase synchronization between all the network nodes is observed, where, in particular, the phase differences between a pair of leaves is zero. The bifurcation analysis reveals two different transitions towards this synchronized state. One occurs through a Hopf bifurcation from a stable equilibrium point. The second occurs from a region of parameters in which the system is not fully ordered. However, this does not exclude some form of partial order such as remote synchronization, which indeed has been numerically and experimentally [Bergner et al., 2012] verified in that region of parameters.

The rest of the paper is organized as follows: the model is introduced in Sec. 2; the theoretical analysis is presented in Sec. 3; some numerical results are discussed in Sec. 4; the conclusions are drawn in Sec. 5 . 


\section{Model}

The dynamics of the star network investigated in this paper is described by the following equations

$$
\begin{gathered}
\dot{x}_{1}=\alpha x_{1}-\omega_{1} y_{1}-x_{1}\left(x_{1}^{2}+y_{1}^{2}\right) \\
+\frac{\lambda}{K} \sum_{i=2}^{N}\left(x_{i}-x_{1}\right) \\
\dot{y}_{1}=\omega_{1} x_{1}+\alpha y_{1}-y_{1}\left(x_{1}^{2}+y_{1}^{2}\right) \\
+\frac{\lambda}{K} \sum_{i=2}^{N}\left(y_{i}-y_{1}\right)
\end{gathered}
$$

and

$$
\begin{gathered}
\dot{x}_{i}=\alpha x_{i}-\omega_{i} y_{i}-x_{i}\left(x_{i}^{2}+y_{i}^{2}\right)+\lambda\left(x_{i}-x_{1}\right) \\
\dot{y}_{i}=\omega_{i} x_{i}+\alpha y_{i}-y_{i}\left(x_{i}^{2}+y_{i}^{2}\right)+\lambda\left(y_{i}-y_{1}\right)
\end{gathered}
$$

with $i=2, \ldots, N$. $\alpha$ is a parameter controlling how fast the trajectory decays onto the attractor, $\omega_{i}$ is the natural frequency of each oscillator, and $\lambda$ is the coupling strength. The constant $K=N-1$ is introduced to simplify the notation.

We define the first oscillator (1) as the $h u b$ of the network and $K$ others (2) as the leaves. We assume that the leaves have the same natural frequency $\omega_{i}=\omega \forall i=2, \ldots, N$, while the hub has a natural frequency $\omega_{1}$, which in general, is different from that of the leaves. The star network studied in this work is schematically represented in Fig. 1.

In polar coordinates, Eqs. (1) and (2) become:

$$
\begin{aligned}
& \dot{\rho}_{1}=\alpha \rho_{1}-\rho_{1}^{3}+\frac{\lambda}{K} \sum_{i=2}^{N}\left(\rho_{i} \cos \left(\theta_{i}-\theta_{1}\right)-\rho_{1}\right) \\
& \dot{\theta}_{1}=\omega_{1}+\frac{\lambda}{K} \sum_{i=2}^{N} \frac{\rho_{1}}{\rho_{i}} \sin \left(\theta_{i}-\theta_{1}\right)
\end{aligned}
$$

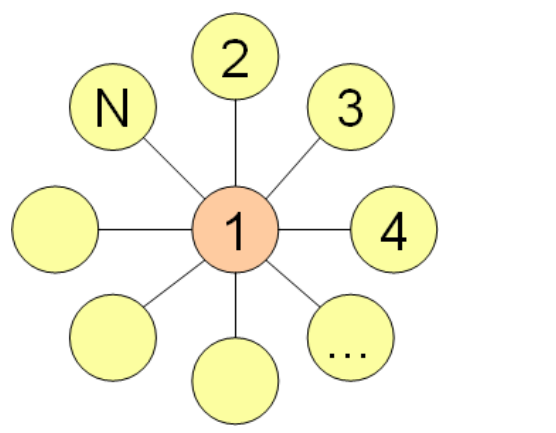

Fig. 1. Schematic representation of a star network. and

$$
\begin{aligned}
& \dot{\rho}_{i}=\alpha \rho_{i}-\rho_{i}^{3}+\lambda\left(\rho_{1} \cos \left(\theta_{1}-\theta_{i}\right)-\rho_{i}\right) \\
& \dot{\theta}_{i}=\omega_{i}+\lambda \frac{\rho_{i}}{\rho_{1}} \sin \left(\theta_{1}-\theta_{i}\right) .
\end{aligned}
$$

In the following, to analyze the behavior of the system, either Eqs. (1) and (2) or Eqs. (3) and (4) will be considered.

\section{Analysis of the Model}

We start the analysis of the model by showing a property of the Laplacian of star networks that is useful for the study of the stability of the system equilibrium point. The stability of this equilibrium point is then analyzed. Then, a necessary condition to obtain synchronization is derived. Finally, we derive and discuss the bifurcation diagram for the system by combining the results of the analysis of stability of the equilibrium point (EP) and the synchronization condition.

Proposition 1. Consider the Laplacian of a star network

$$
\mathrm{G}=\left[\begin{array}{rcccc}
1 & -\frac{1}{K} & -\frac{1}{K} & \cdots & -\frac{1}{K} \\
-1 & 1 & 0 & \cdots & 0 \\
-1 & 0 & 1 & \cdots & 0 \\
\vdots & \vdots & & & \vdots \\
-1 & 0 & 0 & \cdots & 1
\end{array}\right]
$$

and let $\mathrm{T}$ be the diagonalizing matrix such that:

$$
\mathrm{T}^{-1} \mathrm{GT}=\left[\begin{array}{ccccc}
\gamma_{1} & & & & \\
& \gamma_{2} & & & \\
& & \gamma_{3} & & \\
& & & \ddots & \\
& & & & \gamma_{N}
\end{array}\right]
$$

with $\gamma_{1}=2, \gamma_{2}=0$ and $\gamma_{i}=1 \forall i \geq 3, \ldots, N$. Then, if

$$
\mathrm{H}=\left[\begin{array}{lllll}
A & & & & \\
& B & & & \\
& & B & & \\
& & & \ddots & \\
& & & & B
\end{array}\right]
$$


with $A, B \in \mathbb{R}$, the following holds:

$$
\mathrm{T}^{-1} \mathrm{HT}=\left[\begin{array}{ccccc}
\frac{A+B}{2} & \frac{A-B}{2} & & & \\
\frac{A-B}{2} & \frac{A+B}{2} & & & \\
& & B & & \\
& & & \ddots & \\
& & & & B
\end{array}\right] .
$$

Proof. The proof proceeds by direct calculation of the matrix T, its inverse and then Eq. (7).

Matrix $\mathrm{T}$ is constructed as

$$
\mathrm{T}=\left[\begin{array}{lllll}
v^{1} & v^{2} & v^{3} & \cdots & v^{N}
\end{array}\right],
$$

where $v^{1}, v^{2}, v^{3}, \ldots, v^{N}$ are the eigenvectors associated to $\gamma_{1}, \gamma_{2}, \gamma_{3}, \ldots, \gamma_{N}$, respectively. The eigenvectors are calculated by solving the linear system $\left(\mathrm{G}-\gamma_{i} \mathrm{I}\right) v^{i}=0$. For $\gamma_{1}=2$, one gets:

$$
\left\{\begin{array}{l}
-v_{1}^{1}-\frac{1}{K} v_{2}^{1}-\cdots-\frac{1}{K} v_{N}^{1}=0 \\
-v_{1}^{1}-v_{2}^{1}=0 \\
-v_{1}^{1}-v_{3}^{1}=0 \\
\vdots \\
-v_{1}^{1}-v_{N}^{1}=0
\end{array}\right.
$$

and thus $v^{1}=\left[\begin{array}{lllll}1 & -1 & -1 & \cdots & -1\end{array}\right]^{T} \cdot \gamma_{2}=0$ yields:

$$
\left\{\begin{array}{l}
v_{1}^{2}-\frac{1}{K} v_{2}^{2}-\cdots-\frac{1}{K} v_{N}^{2}=0 \\
-v_{1}^{2}+v_{2}^{2}=0 \\
-v_{1}^{2}+v_{3}^{2}=0 \\
\vdots \\
-v_{1}^{2}+v_{N}^{2}=0
\end{array}\right.
$$

and thus $v^{2}=\left[\begin{array}{lllll}1 & 1 & 1 & \cdots & 1\end{array}\right]^{T}$. For $\gamma_{i}=1$ with $i \geq 3, \ldots, N$, one obtains:

$$
\left\{\begin{array}{c}
-\frac{1}{K} v_{2}^{i}-\cdots-\frac{1}{K} v_{N}^{i}=0 \\
v_{1}^{i}=0 \\
\vdots \\
v_{1}^{i}=0
\end{array}\right.
$$

and thus

$$
\begin{aligned}
v^{3} & =\left[\begin{array}{llllll}
0 & 1 & -1 & 0 & \cdots & 0
\end{array}\right]^{T}, \\
v^{4} & =\left[\begin{array}{llllll}
0 & 1 & 0 & -1 & \cdots & 0
\end{array}\right]^{T}
\end{aligned}
$$

and so on $v^{N}=\left[\begin{array}{llllll}0 & 1 & 0 & 0 & \cdots & -1\end{array}\right]^{T}$. Matrix T is therefore given by:

$$
\mathrm{T}=\left[\begin{array}{rrrrr}
1 & 1 & 0 & \cdots & 0 \\
-1 & 1 & 1 & \cdots & 1 \\
-1 & 1 & -1 & \cdots & 0 \\
\vdots & \vdots & \vdots & \ddots & \vdots \\
-1 & 1 & 0 & \cdots & -1
\end{array}\right] .
$$

The determinant of $\mathrm{T}$ is given by: $\operatorname{det} \mathrm{T}=$ $2(-1)^{N}(N-1)$, while its inverse is:

$$
\mathrm{T}^{-1}=\left[\begin{array}{ccccc}
\frac{1}{2} & -\frac{1}{2(N-1)} & -\frac{1}{2(N-1)} & \cdots & -\frac{1}{2(N-1)} \\
\frac{1}{2} & \frac{1}{2(N-1)} & \frac{1}{2(N-1)} & \cdots & \frac{1}{2(N-1)} \\
0 & \frac{1}{N-1} & -\frac{N-2}{N-1} & \cdots & \frac{1}{N-1} \\
\vdots & \vdots & \vdots & \ddots & \vdots \\
0 & \frac{1}{N-1} & \frac{1}{N-1} & \cdots & -\frac{N-2}{N-1}
\end{array}\right] .
$$


Finally, let us compute $\mathrm{T}^{-1} \mathrm{HT}$ as

$\mathrm{T}^{-1} \mathrm{HT}$

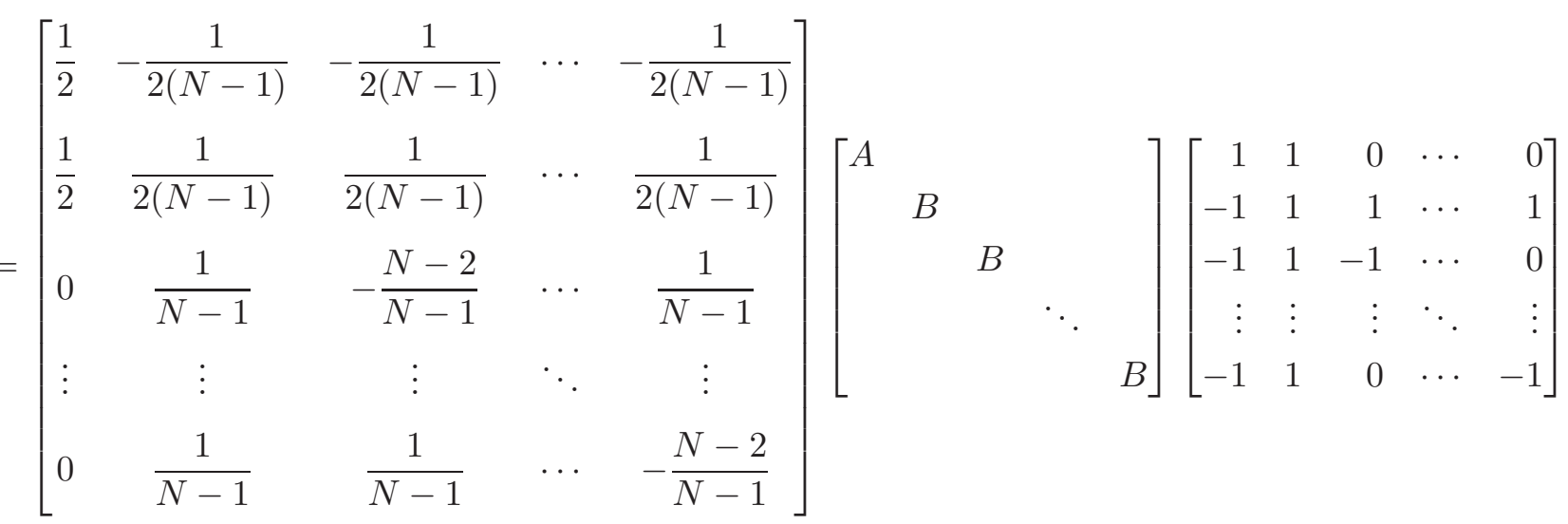$$
=\left[\begin{array}{ccccc}
\frac{A}{2} & -\frac{B}{2(N-1)} & -\frac{B}{2(N-1)} & \cdots & -\frac{B}{2(N-1)} \\
\frac{A}{2} & \frac{B}{2(N-1)} & \frac{B}{2(N-1)} & \cdots & \frac{B}{2(N-1)} \\
0 & \frac{B}{N-1} & -\frac{N-2}{N-1} B & \cdots & \frac{B}{N-1} \\
\vdots & \vdots & \vdots & \ddots & \vdots \\
0 & \frac{B}{N-1} & \frac{B}{N-1} & \cdots & -\frac{N-2}{N-1} B
\end{array}\right]\left[\begin{array}{rrrrr}
1 & 1 & 0 & \cdots & 0 \\
-1 & 1 & 1 & \cdots & 1 \\
-1 & 1 & -1 & \cdots & 0 \\
\vdots & \vdots & \vdots & \ddots & \vdots \\
-1 & 1 & 0 & \cdots & -1
\end{array}\right]
$$$$
=\left[\begin{array}{lllll}
\frac{A+B}{2} & \frac{A-B}{2} & & & \\
\frac{A-B}{2} & \frac{A+B}{2} & & & \\
& & B & & \\
& & & \ddots & \\
& & & & B
\end{array}\right]
$$

which completes the proof.

Proposition 1 can be easily generalized to block matrices, i.e. if

$$
\mathrm{H}=\left[\begin{array}{lllll}
\mathrm{A} & & & & \\
& \mathrm{B} & & & \\
& & \mathrm{B} & & \\
& & & \ddots & \\
& & & & \mathrm{B}
\end{array}\right]
$$

with $\mathrm{A}, \mathrm{B} \in \mathbb{R}^{n \times n}$, the following holds:

$$
\mathrm{T}^{-1} \otimes \mathrm{I}_{n} \cdot \mathrm{H} \cdot \mathrm{T} \otimes \mathrm{I}_{n}
$$

$$
=\left[\begin{array}{ccccc}
\frac{\mathrm{A}+\mathrm{B}}{2} & \frac{\mathrm{A}-\mathrm{B}}{2} & & & \\
\frac{\mathrm{A}-\mathrm{B}}{2} & \frac{\mathrm{A}+\mathrm{B}}{2} & & & \\
& & \mathrm{~B} & & \\
& & & \ddots & \\
& & & & \mathrm{B}
\end{array}\right]
$$


where $\mathrm{I}_{n}$ is the $n \times n$ identity matrix. This property is now used for the study of the stability of the equilibrium point of systems (1) and (2). In fact, this system has one equilibrium point, usually named oscillation death (or quenching), given by $x_{i}=y_{i}=0 \quad \forall i=1, \ldots, N$. Incidentally, we note that oscillation or amplitude death has been observed in many different topologies of networks of Stuart-Landau oscillators [Ermentrout, 1990;
Mirollo \& Strogatz, 1990; Nakao \& Mikhailov, 2009; Liu et al., 2009]. The stability properties of this equilibrium point can be derived by studying the system Jacobian and the associated eigenvalues. In fact, these eigenvalues can be analytically calculated as shown in the following proposition.

Proposition 2. The eigenvalues of the Jacobian of systems (1) and (2) around the equilibrium point $x_{i}=y_{i}=0 \forall i=1, \ldots, N$ are given by:

$$
\begin{aligned}
& \mu_{1,2,3,4}=\alpha-\lambda \pm \sqrt{4 \lambda^{2}-2 \omega_{1}^{2}-2 \omega^{2} \pm 2 \sqrt{\left(\omega_{1}+\omega\right)^{2}\left(\omega_{1}^{2}+\omega^{2}-2 \omega \omega_{1}-4 \lambda^{2}\right)}} \\
& \mu_{5, \ldots, 2 N}=\alpha-\lambda \pm j \omega .
\end{aligned}
$$

Proof. The Jacobian of the system around the equilibrium point $x_{i}=y_{i}=0 \forall i=1, \ldots, N$ is given by:

$$
\mathrm{J}=\left[\begin{array}{ccccccc}
\alpha-\lambda & -\omega_{1} & \frac{\lambda}{K} & 0 & \cdots & \frac{\lambda}{K} & 0 \\
\omega_{1} & \alpha-\lambda & 0 & \frac{\lambda}{K} & \cdots & 0 & \frac{\lambda}{K} \\
\lambda & 0 & \alpha-\lambda & -\omega & \cdots & 0 & 0 \\
0 & \lambda & \omega & \alpha-\lambda & \cdots & 0 & 0 \\
\vdots & \vdots & & & & & \vdots \\
\lambda & 0 & 0 & 0 & \cdots & \alpha-\lambda & -\omega \\
0 & \lambda & 0 & 0 & \cdots & \omega & \alpha-\lambda
\end{array}\right] .
$$

If we consider the Laplacian of the star network

$$
\mathrm{G}=\left[\begin{array}{rcccc}
1 & -\frac{1}{K} & -\frac{1}{K} & \cdots & -\frac{1}{K} \\
-1 & 1 & 0 & \cdots & 0 \\
-1 & 0 & 1 & \cdots & 0 \\
\vdots & \vdots & \vdots & & \vdots \\
-1 & 0 & 0 & \cdots & 1
\end{array}\right]
$$

the Jacobian can be expressed in a more compact form:

$$
\mathrm{J}=\left[\begin{array}{cccc}
\mathrm{J}_{1} & 0 & \cdots & 0 \\
0 & \mathrm{~J}_{2} & \cdots & 0 \\
\vdots & \vdots & & \vdots \\
0 & 0 & \cdots & \mathrm{J}_{2}
\end{array}\right]-\lambda \mathrm{G} \otimes \mathrm{I}_{2}
$$

where $\mathrm{J}_{1}=\left[\begin{array}{cc}\alpha & -\omega_{1} \\ \omega_{1} & \alpha\end{array}\right], \mathrm{J}_{2}=\left[\begin{array}{cc}\alpha & -\omega \\ \omega & \alpha\end{array}\right]$ and $\mathrm{I}_{2}=\left[\begin{array}{ll}1 & 0 \\ 0 & 1\end{array}\right]$.

To calculate the eigenvalues of $\mathrm{J}$, the property of the Laplacian of the star network, discussed above, is used. According to this, it can be shown that:

$$
\begin{aligned}
& \mathrm{T}^{-1} \otimes \mathrm{I}_{2} \cdot \mathrm{J} \cdot \mathrm{T} \otimes \mathrm{I}_{2} \\
& =\left[\begin{array}{ccccc}
\frac{\mathrm{J}_{1}+\mathrm{J}_{2}}{2} & \frac{\mathrm{J}_{1}-\mathrm{J}_{2}}{2} & & & \\
\frac{\mathrm{J}_{1}-\mathrm{J}_{2}}{2} & \frac{\mathrm{J}_{1}+\mathrm{J}_{2}}{2} & & & \\
& & \mathrm{~J}_{2} & & \\
& & \ddots & \\
& & & & \mathrm{J}_{2}
\end{array}\right] \\
& -\lambda\left[\begin{array}{ccccc}
\gamma_{1} & & & & \\
& \gamma_{2} & & & \\
& & \gamma_{3} & & \\
& & & \ddots & \\
& & & & \gamma_{N}
\end{array}\right] \otimes \mathrm{I}_{2}
\end{aligned}
$$


where $\gamma_{1}=2, \gamma_{2}=0, \gamma_{3}=\cdots=\gamma_{N}=1$ are the eigenvalues of $\mathrm{G}$. Since the matrices $\tilde{\mathrm{J}} \triangleq \mathrm{T}^{-1} \otimes \mathrm{I}_{2} \cdot \mathrm{J} \cdot \mathrm{T} \otimes \mathrm{I}_{2}$ and $\mathrm{J}$ have the same eigenvalues, it can be concluded that the eigenvalues of $\mathrm{J}$ are given by the eigenvalues of $\mathrm{J}_{2}=\left[\begin{array}{cc}\alpha & -\omega \\ \omega & \alpha\end{array}\right]$ with multiplicity $(N-2)$ and by those of the $4 \times 4$ block:

$$
\mathrm{J}_{p}=\left[\begin{array}{cccc}
\alpha-2 \lambda & -\frac{\omega_{1}+\omega}{2} & 0 & -\frac{\omega_{1}-\omega}{2} \\
\frac{\omega_{1}+\omega}{2} & \alpha-2 \lambda & \frac{\omega_{1}-\omega}{2} & 0 \\
0 & -\frac{\omega_{1}-\omega}{2} & \alpha & -\frac{\omega+\omega_{1}}{2} \\
\frac{\omega_{1}-\omega}{2} & 0 & \frac{\omega+\omega_{1}}{2} & \alpha
\end{array}\right]
$$

which are

$$
\mu_{1,2,3,4}=\alpha-\lambda \pm \sqrt{4 \lambda^{2}-2 \omega_{1}^{2}-2 \omega^{2} \pm 2 \sqrt{\left(\omega_{1}+\omega\right)^{2}\left(\omega_{1}^{2}+\omega^{2}-2 \omega \omega_{1}-4 \lambda^{2}\right)}}
$$

In summary, the eigenvalues of $\mathrm{J}$ are given by:

$$
\begin{aligned}
& \mu_{1,2,3,4}=\alpha-\lambda \pm \sqrt{4 \lambda^{2}-2 \omega_{1}^{2}-2 \omega^{2} \pm 2 \sqrt{\left(\omega_{1}+\omega\right)^{2}\left(\omega_{1}^{2}+\omega^{2}-2 \omega \omega_{1}-4 \lambda^{2}\right)}} \\
& \mu_{5, \ldots, 2 N}=\alpha-\lambda \pm j \omega .
\end{aligned}
$$

We now study the stability of the equilibrium point $x_{i}=y_{i}=0 \forall i=1, \ldots, N$. The system admits a stable equilibrium point when the real part of all the eigenvalues is negative. Since $\Re\left[\mu_{5, \ldots, 2 N}\right]=\alpha-\lambda$, the eigenvalues $\mu_{5, \ldots, 2 N}$ have negative real part for $\lambda>\alpha$. To study the sign of the eigenvalues $\mu_{1,2,3,4}$, we consider the characteristic polynomial of $\mathrm{J}_{p}$ :

$$
\begin{aligned}
\Phi(p) & =\operatorname{det}\left(p \mathrm{I}-\mathrm{J}_{p}\right) \\
& =p^{4}+A p^{3}+B p^{2}+C p+D
\end{aligned}
$$

with

$$
\begin{aligned}
A= & 4 \lambda-4 \alpha \\
B= & 4 \lambda^{2}+\omega_{1}^{2}+6 \alpha^{2}+\omega^{2}-12 \lambda \alpha \\
C= & -2 \alpha \omega^{2}-4 \alpha^{3}-8 \lambda^{2} \alpha-2 \alpha \omega_{1}^{2}+2 \lambda \omega_{1}^{2} \\
& +2 \lambda \omega^{2}+12 \lambda \alpha^{2} \\
D= & -2 \alpha \lambda \omega^{2}+\lambda^{2} \omega^{2}+\lambda^{2} \omega_{1}^{2}+\omega^{2} \omega_{1}^{2} \\
& +2 \lambda^{2} \omega \omega_{1}+\alpha^{4}+\alpha^{2} \omega_{1}^{2}-4 \lambda \alpha^{3} \\
& -2 \alpha \lambda \omega_{1}^{2}+\alpha^{2} \omega^{2}+4 \lambda^{2} \alpha^{2}
\end{aligned}
$$

$\Phi(p)$ admits a solution with zero imaginary part if and only if:

$$
C^{2}-B C A+D A^{2}=0
$$

Solving Eq. (22) for $\lambda$, one finds that at least one of the two pairs $\mu_{1,2,3,4}$ crosses the imaginary axis if $\lambda=\frac{1}{8 \alpha}\left(4 \alpha^{2}+\Delta \omega^{2}\right)$, where $\Delta \omega=\omega-\omega_{1}$. Finally, studying the sign of the real part of $\mu_{1,2,3,4}$, it can be concluded that the equilibrium point is stable for $\lambda>\alpha$ and $\lambda<\frac{1}{8 \alpha}\left(4 \alpha^{2}+\Delta \omega^{2}\right)$, which, in the $\Delta \omega-\lambda$ plane, leads to the red region shown in Fig. 2.

It is important to note that, assuming that $K \geq 2$, the result does not depend on the number of leaves $K$.

A necessary condition to obtain synchronization in the system of coupled Stuart-Landau oscillators is now derived.

Proposition 3. A necessary condition for systems (1) and (2) to be synchronized is that:

$$
\lambda \geq \frac{|\Delta \omega|}{2} .
$$




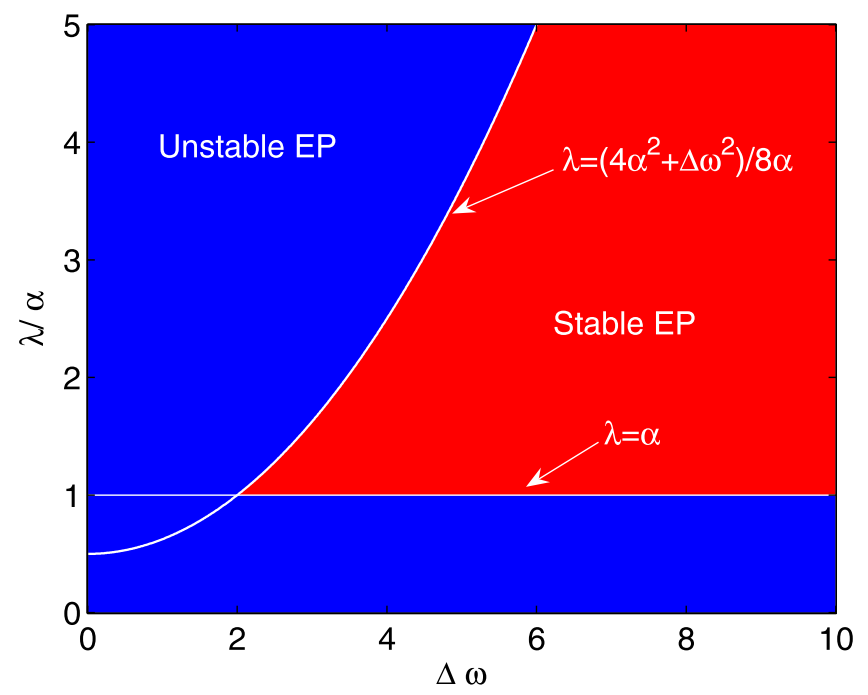

Fig. 2. Stability of the equilibrium point (EP) $x_{i}=y_{i}=0$ for systems (1) and (2).

Proof. For convenience we start from the system written in polar coordinates as in Eqs. (3) and (4). In polar coordinates, phase synchronization is characterized by constant amplitudes $\left(\dot{\rho}_{i}=0 \forall i=\right.$ $1, \ldots, N)$ and constant phase difference between oscillators.

Let us first consider the amplitudes. Consider the equilibrium condition $\left(\dot{\rho}_{i}=0 \forall i=1, \ldots, N\right)$, multiply the first equation of (3) by $K$ and subtract the first equations of (4) for $i=2, \ldots, N$ to obtain:

$$
\begin{gathered}
K\left(\alpha \rho_{1}-\rho_{1}^{3}\right)+\lambda \sum_{i=2}^{N}\left(\rho_{i} \cos \left(\theta_{i}-\theta_{1}\right)-\rho_{1}\right) \\
-\sum_{i=2}^{N}\left(\alpha \rho_{i}-\rho_{i}^{3}\right) \\
-\lambda \sum_{i=2}^{N}\left(\rho_{1} \cos \left(\theta_{1}-\theta_{i}\right)-\rho_{i}\right)=0 .
\end{gathered}
$$

From this, we can derive that $\rho_{1}=\rho_{i}$, i.e. at equilibrium (network synchronization) the amplitudes of the oscillations at each node are the same.

Let us now consider the equations for the phases. We assume that the Stuart-Landau oscillators are isochronous and define $\Omega$ as the common frequency of oscillations of all the nodes of the network when synchronized. Let us then introduce a set of new variables $\zeta_{i}$ defined as $\zeta_{i}=\theta_{i}-\Omega t$ and, taking into account that at the equilibrium $\rho_{1}=\rho_{i} \forall i=2, \ldots, N$, rewrite systems (3) and (4) as follows:

$$
\dot{\zeta}_{1}=\omega_{1}-\Omega+\frac{\lambda}{K} \sum_{i=2}^{N} \sin \left(\zeta_{i}-\zeta_{1}\right)
$$

and

$$
\dot{\zeta}_{i}=\omega_{i}-\Omega+\lambda \sin \left(\zeta_{1}-\zeta_{i}\right)
$$

At the equilibrium the phase difference between oscillators is constant (i.e. $\dot{\zeta}_{i}=0 \forall i=1, \ldots, N$ ), so we get (for the hub):

$$
\omega_{1}-\Omega+\frac{\lambda}{K} \sum_{i=2}^{N} \sin \left(\zeta_{i}-\zeta_{1}\right)=0
$$

and for the leaves:

$$
\omega_{i}-\Omega+\lambda \sin \left(\zeta_{1}-\zeta_{i}\right)=0
$$

From these equations, we first derive $\Omega$. Multiply (27) by $K$, sum Eq. (28) for $i=2, \ldots, N$ and sum the two results to obtain:

$$
K\left(\omega_{1}-\Omega\right)+\sum_{i=2}^{N}\left(\omega_{i}-\Omega\right)=0 .
$$

Taking into account that $\omega_{i}=\omega, \Omega$ is thus given by $\Omega=\frac{\omega_{1}+\omega}{2}$. Therefore, when all the nodes of the network are synchronized, they will oscillate at $\Omega=\frac{\omega_{1}+\omega}{2}$.

Let us now consider the phase equation at the equilibrium for one of the leaves of the system:

$$
\omega_{i}-\Omega+\lambda \sin \left(\zeta_{1}-\zeta_{i}\right)=0
$$

and thus

$$
\sin \left(\zeta_{1}-\zeta_{i}\right)=\frac{\omega_{1}-\omega}{2 \lambda}
$$

At equilibrium, $\zeta_{1}-\zeta_{i}$ is constant. A stable solution of Eq. (31) exists if

$$
\frac{\left|\omega_{1}-\omega\right|}{2 \lambda} \leq 1
$$

and thus if

$$
\lambda \geq \frac{|\Delta \omega|}{2}
$$

Based on the stability analysis reported above and from the results of Proposition 3, the bifurcation diagram of systems (1) and (2) as shown in Fig. 3 can be derived. Three different regions can be distinguished: stable equilibrium point (red region); synchronization (yellow region) and disorder (blue region). The term disorder here refers to 


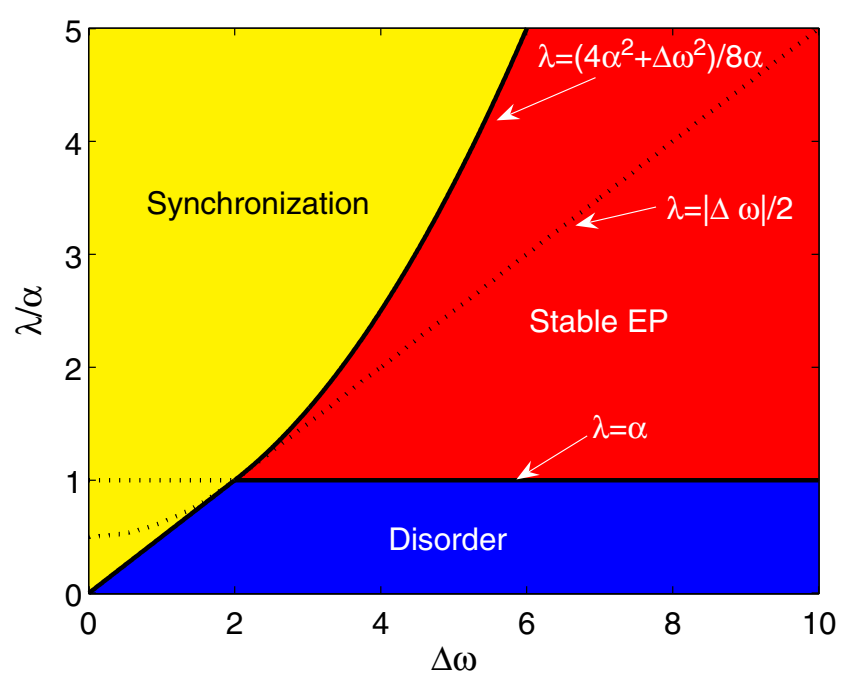

Fig. 3. Bifurcation diagram for systems (1) and (2).

a not fully ordered system, but does not exclude the possibility of some partially ordered state (as it will be shown in the following, in fact, remote synchronization occurs in this region).

The synchronization region (Arnold tongue) is characterized by $\lambda \geq \frac{\alpha^{2}+\Delta \omega^{2}}{8 \alpha}$ for $\lambda \geq \alpha$ and by $\lambda \geq \frac{|\Delta \omega|}{2}$ for $\lambda<\alpha$. It is interesting to note that the transition to synchronization occurs in two different ways. If $\Delta \omega>2 \alpha$ and $\lambda$ is increased starting from zero, first the system reaches a regime characterized by a stable equilibrium point. Then, if $\lambda$ is further increased until the line $\lambda=\frac{\alpha^{2}+\Delta \omega^{2}}{8 \alpha}$ is crossed, a Hopf bifurcation leads the system to a regime of synchronized behavior. In this case, indicated as $\lambda_{c}$ the bifurcation point $\left(\lambda_{c}=\frac{4 \alpha^{2}+\Delta \omega^{2}}{8 \alpha}\right)$ the amplitude of synchronized oscillations is proportional to $\left(\lambda-\lambda_{c}\right)^{1 / 2}$. Instead, when $\Delta \omega<2 \alpha$ and $\lambda$ is increased starting from zero, the system starts in the disorder region and then synchronization occurs when the line $\lambda=\frac{|\Delta \omega|}{2}$ is crossed. The first kind of transition excludes the possibility of a transition through remote synchronization, while, in the second case, a transition through remote synchronization has been both numerically and experimentally observed [Bergner et al., 2012].

\section{Numerical Results}

An example of Eqs. (1) and (2) with $N=6, \omega=1.1$ and $\alpha=2$ is considered in this section. We first set $\omega_{1}=3$, i.e. $|\Delta \omega|=1.9$. According to the analysis
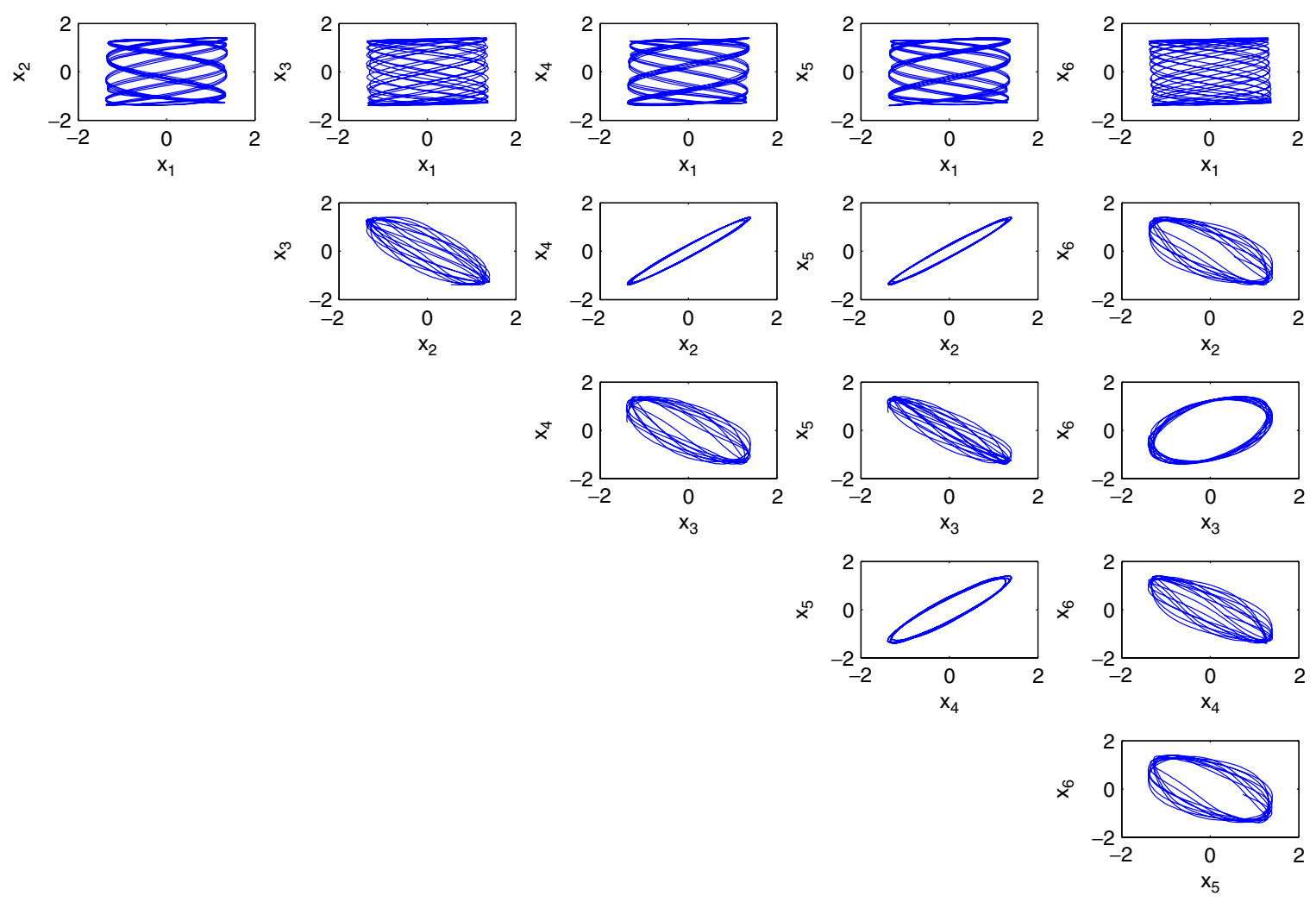

Fig. 4. Phase diagrams $x_{1}^{i}-x_{1}^{j}$ for systems (1) and (2) with $N=6, \alpha=2, \omega=1.1, \omega_{1}=3$ and $\lambda=0.2$. 
M. Frasca et al.
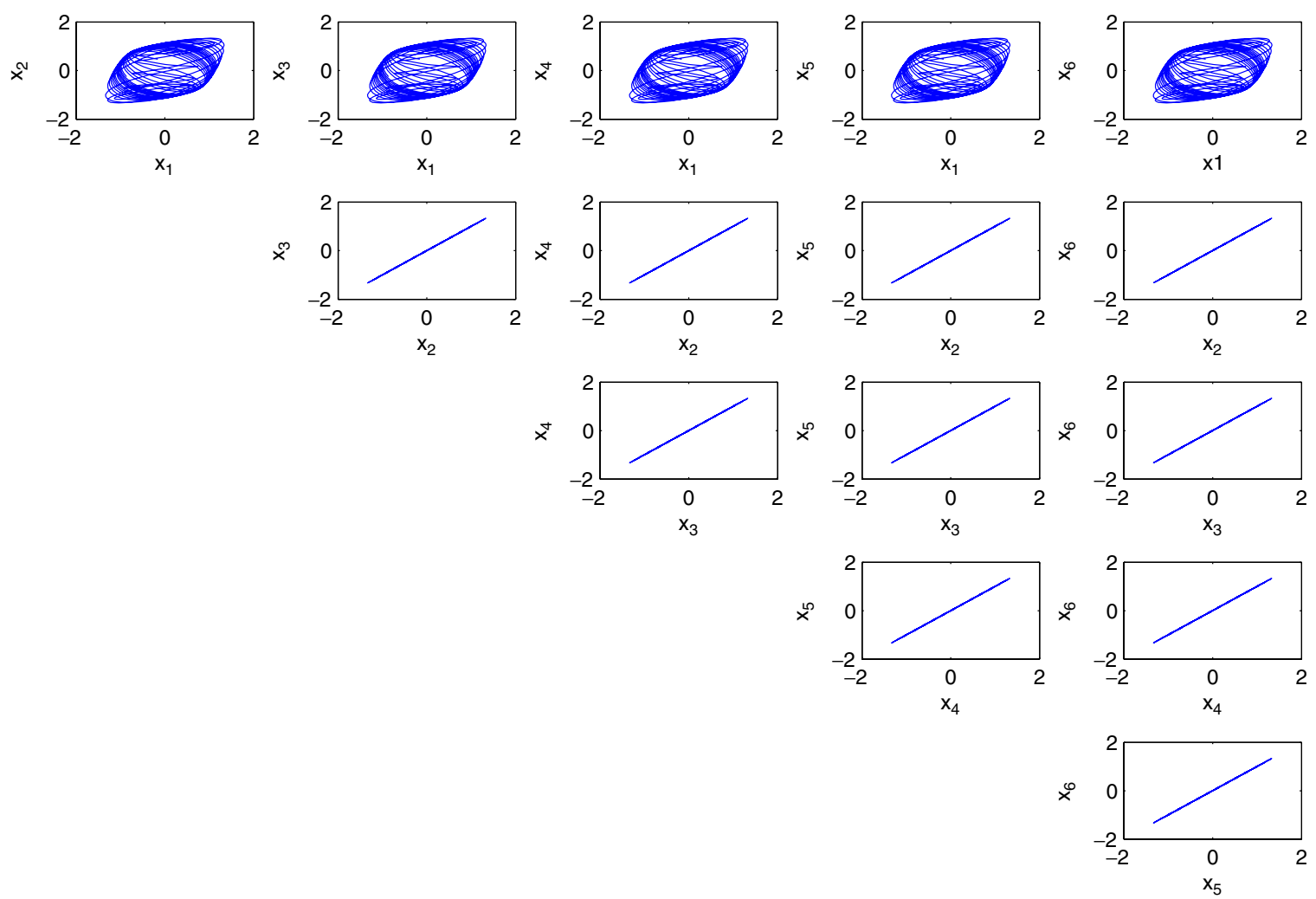

Fig. 5. Phase diagrams $x_{1}^{i}-x_{1}^{j}$ for systems (1) and (2) with $N=6, \alpha=2, \omega=1.1, \omega_{1}=3$ and $\lambda=0.9$.
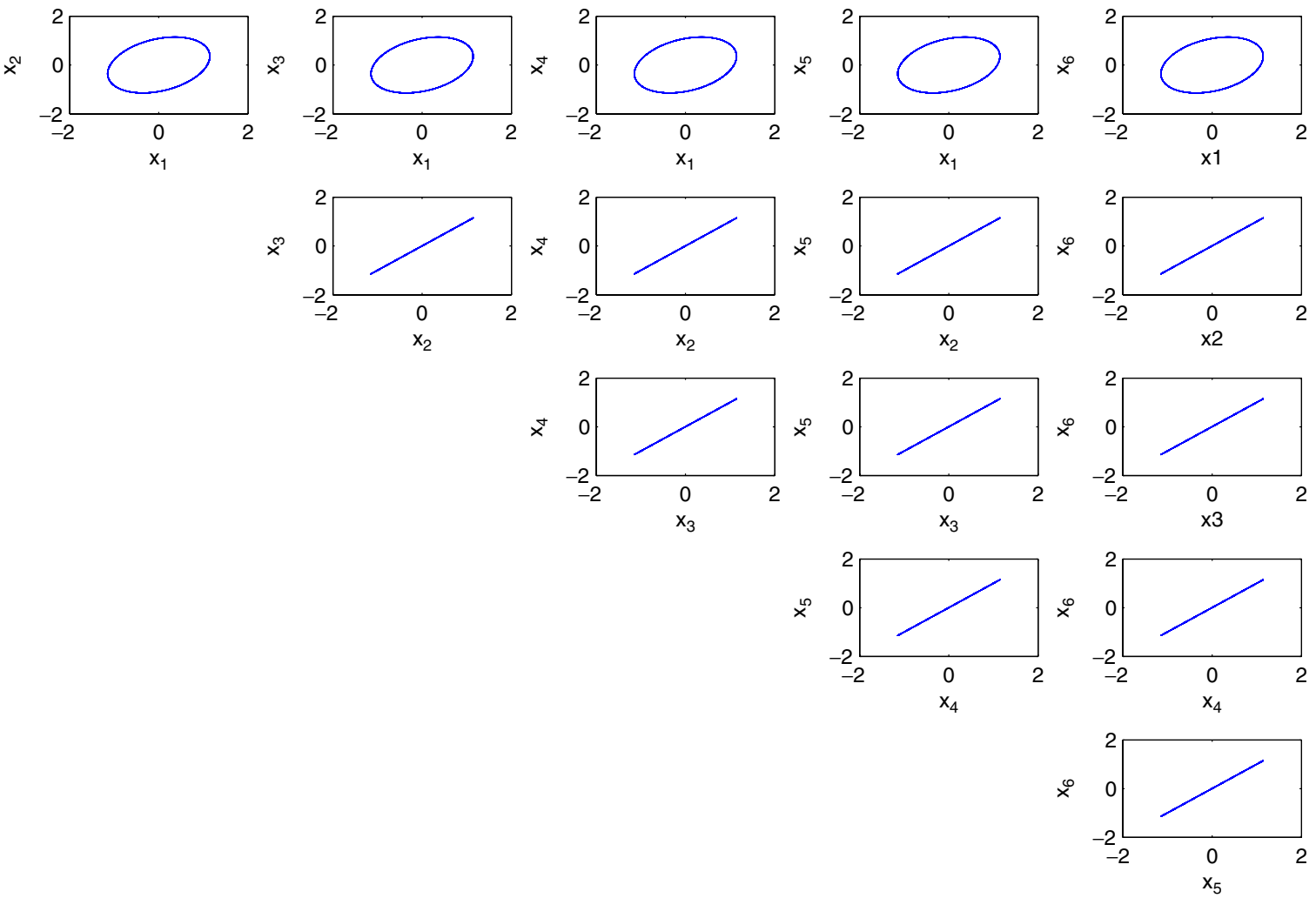

Fig. 6. Phase diagrams $x_{1}^{i}-x_{1}^{j}$ for systems (1) and (2) with $N=6, \alpha=2, \omega=1.1, \omega_{1}=3$ and $\lambda=1.0$. 


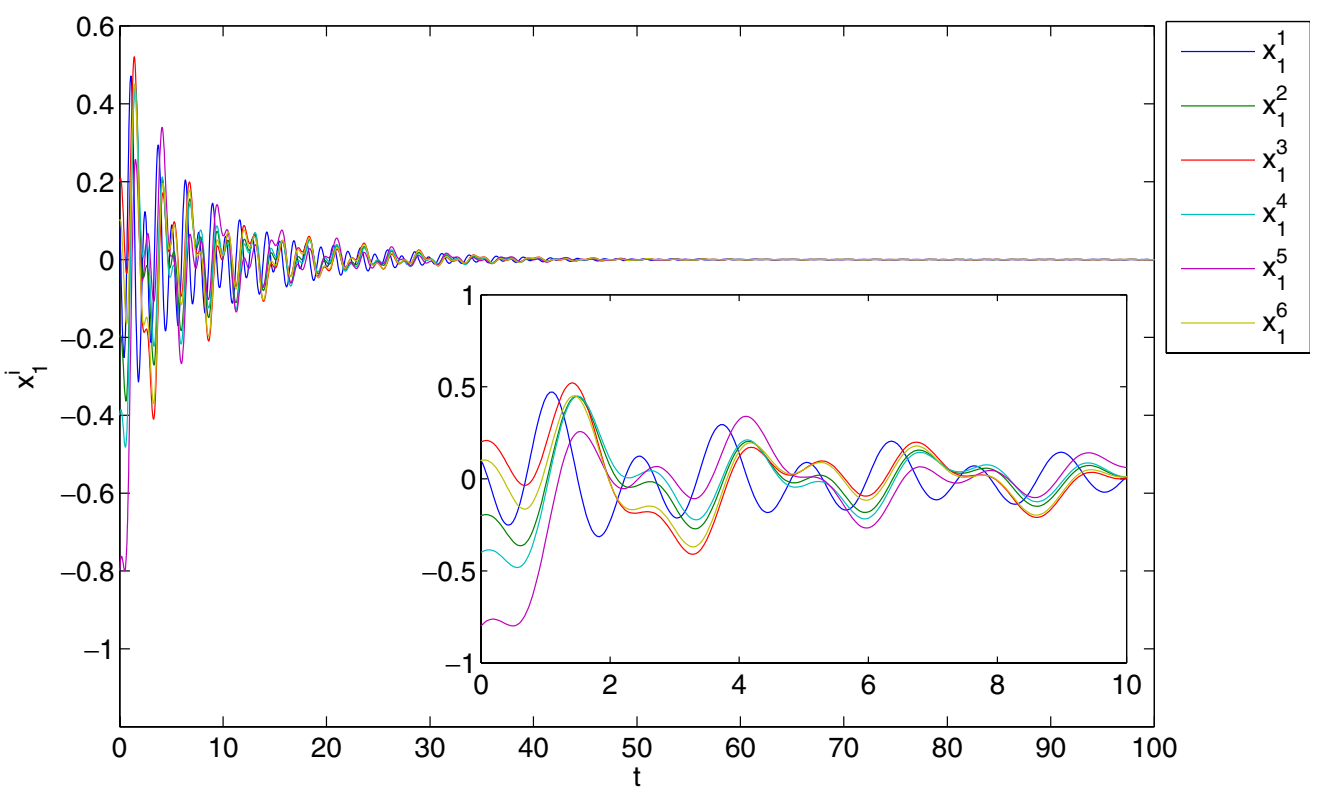

Fig. 7. Trends of $x_{1}^{i}$ for systems (1) and (2) with $N=6, \alpha=2, \omega=1.1, \omega_{1}=6$ and $\lambda=2.1$.

discussed in Sec. 3, the system will be fully synchronized for $\lambda>\frac{|\Delta \omega|}{2}=0.95$. The transition to full synchronization occurs through a weaker form of synchronization, i.e. remote synchronization. In fact, for $\lambda=0.2$ the oscillators are not synchronized (Fig. 4); for $\lambda=0.9$ remote synchronization (i.e. a regime in which the external nodes are synchronized to each other, but the central one is not
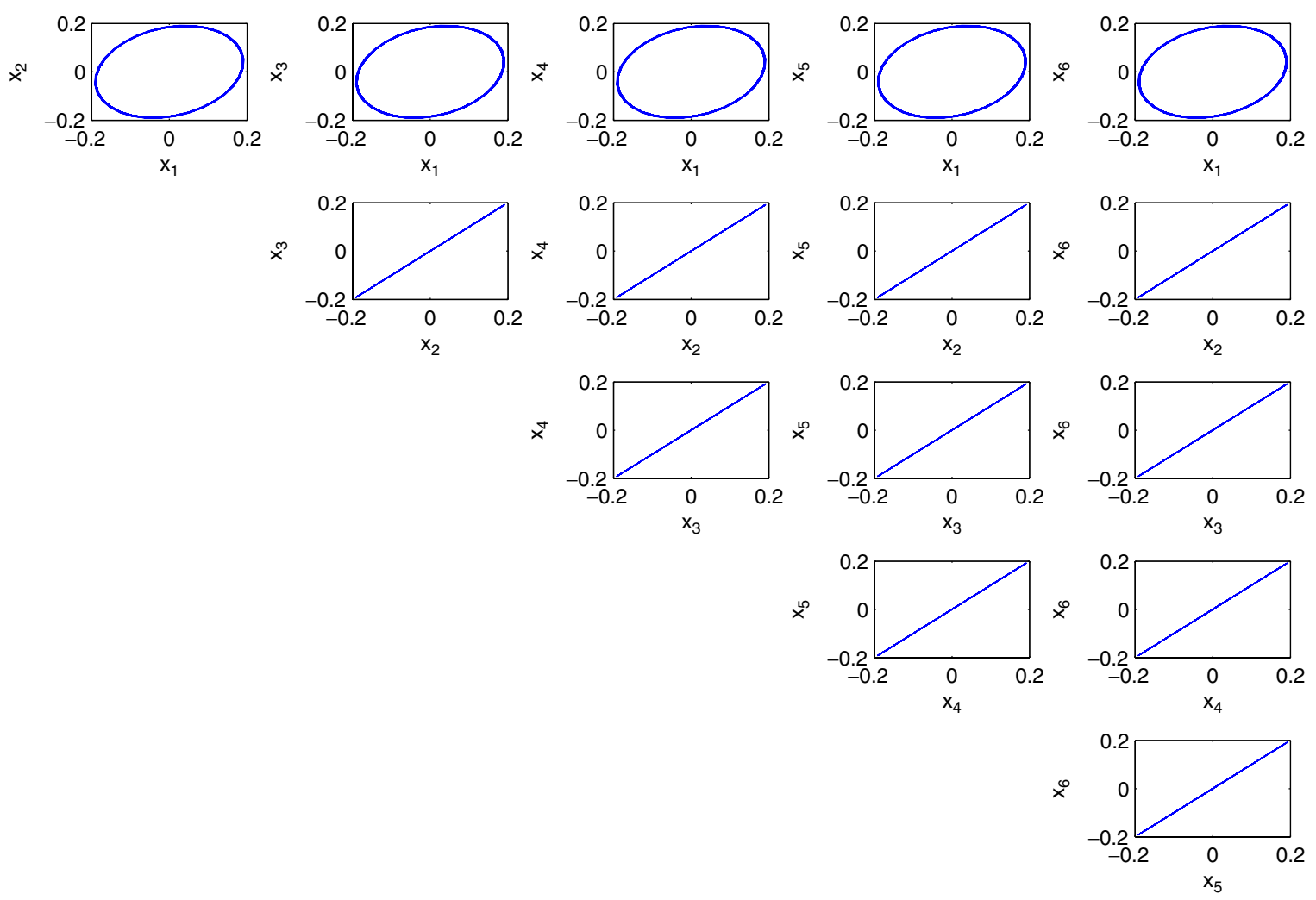

Fig. 8. Phase diagrams $x_{1}^{i}-x_{1}^{j}$ for systems (1) and (2) with $N=6, \alpha=2, \omega=1.1, \omega_{1}=6$ and $\lambda=2.51$. 
synchronized) is observed (Fig. 5); for $\lambda=1$ the oscillators are fully synchronized (Fig. 6).

We then consider $\omega_{1}=6$. For $\lambda>\alpha=2$ the system regime is a stable equilibrium point, as shown in Fig. 7 , where $\lambda$ is set to $\lambda=2.1$. When $\lambda>\lambda_{c}=\frac{4 \alpha^{2}+\Delta \omega^{2}}{8 \alpha}=2.5006$, a Hopf bifurcation occurs. As shown in Fig. 8, obtained for $\lambda=2.51$, the nodes oscillate synchronously with an amplitude proportional to $\left(\lambda-\lambda_{c}\right)^{1 / 2}$.

\section{Conclusions}

In this paper, an analytical study of a star motif of Stuart-Landau oscillators has been presented. The system consists of a central node, the hub, connected to an arbitrary number of peripheral nodes, the leaves (the analysis discussed does not depend on the number of leaves). It is assumed that the leaves have the same parameters, which in general are different from that of the hub. Under these hypotheses, very interesting behaviors can arise in such star network topologies. In particular, in [Bergner et al., 2012] it has been reported that remote synchronization, a regime in which the leaves are synchronized among each other, but the hub is not, may appear. Instead, in [GómezGardeñes et al., 2011] the same topology is studied as a limit case of the more general class of scale-free networks exhibiting explosive synchronization, but the model of the nodes has been assumed to be a Kuramoto purely phase oscillator.

In our work, starting from the analysis of the topological properties of the star configuration, some analytical considerations have been applied to derive the bifurcation diagram of the system with respect to the parameter mismatch between leaves and hub and to the coupling strength. The analysis revealed that the system may become fully synchronized (more precisely, the leaves are completely synchronized among each other and phase synchronized with the hub) through two different transitions. One occurs through a Hopf bifurcation. The second does from a region in which the system is not fully ordered. It is important to note that this second kind of transition exactly matches the transition to global synchronization through remote synchronization as numerically and experimentally discussed in [Bergner et al., 2012]. The phenomenon, apparently paradoxical, of synchronization of not directly connected nodes, occurs thanks to the effect of the modulation of the hub amplitude, and leads to a condition in which a partial form of order is already observed at the transition to synchronization. Since Kuramoto oscillators (analyzed in [Gómez-Gardeñes et al., 2011]) are purely phase oscillators, remote synchronization cannot be observed in such systems, but the existence of a partially ordered state just before the transition to synchronization is reflected on the high value of the Kuramoto order parameter in the explosive synchronization phenomenon revealed in [Gómez-Gardeñes et al., 2011].

Finally, we point out that the properties of the star network topology introduced in our work may find further applications in the analysis of other dynamical systems, especially in the combination with master stability function based approaches, where instead of an exact calculation of the eigenvalues, numerical analyses of the maximum Lyapunov exponents are required.

\section{Acknowledgments}

This work was supported by DAAD/Ateneo ItaloTedesco under the VIGONI Project and DFG Research group HIMPAC and EU ITN LINC.

\section{References}

Afraimovich, V. S., Verichev, N. N. \& Rabinovich, M. I. [1986] "Stochastic synchronization of oscillation in dissipative systems," Radiophys. Quant. Electron. 29, 795-803.

Amriktar, R. E. \& Rangarajan, G. [2009] "Stability of multicluster synchronization," Int. J. Bifurcation and Chaos 19, 4263-4271.

Aoyagi, T. [1995] "Networks of neural oscillators for retrieving phase information," Phys. Rev. Lett. 74, 4075-4078.

Arena, P., Buscarino, A., Fortuna, L. \& Frasca, M. [2006] "Separation and synchronization of piecewise linear chaotic systems," Phys. Rev. E 74, 026212-1-11.

Arenas, A., Diaz-Guilera, A., Kurths, J., Moreno, Y. \& Zhou, C. [2008] "Synchronization in complex networks," Phys. Rep. 469, 93-153.

Bergner, A., Frasca, M., Sciuto, G., Buscarino, A., Ngamga, E., Fortuna, L. \& Kurths, J. [2012] "Remote synchronization in network motifs," Phys. Rev. E 85, 026208-1-7.

Boccaletti, S., Kurths, J., Osipov, G., Valladares, D. L. \& Zhou, C. S. [2002] "The synchronization of chaotic systems," Phys. Rep. 366, 1-101.

Boccaletti, S., Latora, V., Moreno, Y., Chavez, M. \& Hwang, D. U. [2006] "Complex networks: Structure and dynamics," Phys. Rep. 424, 175-308. 
Burwick, T. [2006] "Oscillatory networks: Pattern recognition without a superposition catastrophe," Neural Comput. 18, 356-380.

Buscarino, A., Fortuna, L., Frasca, M. \& Sciuto, G. [2009] "Chua's circuits synchronization with diffusive coupling: New results," Int. J. Bifurcation and Chaos 19, 3101-3107.

Chen, L. \& Lu, J. [2008] "Cluster synchronization in a complex dynamical network with two nonidentical clusters," J. Syst. Sci. Compl. 21, 20-33.

D'Huys, O., Vicente, R., Erneux, T., Danckaert, J. \& Fischer, I. [2008] "Synchronization properties of network motifs: Influence of coupling delay and symmetry," Chaos 18, 037116-1-11.

Ermentrout, G. B. [1990] "Oscillator death in populations of "all to all" coupled nonlinear oscillators," Physica D 41, 219-231.

Fortuna, L. \& Frasca, M. [2007] "Experimental synchronization of single-transistor-based chaotic circuits," Chaos 17, 043118-1-5.

Fujisaka, H. \& Yamada, T. [1983] "Stability theory of synchronized motion in coupled-oscillator systems," Progr. Theoret. Phys. 69, 32-47.

Gómez-Gardeñes, J., Gómez, S., Arenas, A. \& Moreno, Y. [2011] "Explosive synchronization transitions in scale-free networks," Phys. Rev. Lett. 106, 128701.

Hakim, V. \& Rappel, W. J. [1992] "Dynamics of globally coupled complex Ginzburg-Landau equation," Phys. Rev. A 46, 7347-7350.

Hoppensteadt, F. C. \& Izhikevich, E. M. [2001] "Synchronization of MEMS resonators and mechanical neurocomputing," IEEE Trans. Circuits Syst.-I: Fund. Th. Appl. 48, 133-138.

Kawaguchi, S. [2000] "Stability gap between off- and onfiring states in a coupled Ginzburg-Landau oscillator neural network," Progr. Theoret. Phys. 104, 709-721.

Liu, W., Wang, X., Guan, S. \& Lai, C. H. [2009] "Transition to amplitude death in scale-free networks," New J. Phys. 11, 093016.

Lodato, I., Boccaletti, S. \& Latora, V. [2007] "Synchronization properties of network motifs," Europhys. Lett. 78, 28001-1-5.

Matthews, P. C. \& Strogatz, S. H. [1990] "Phase diagram for the collective behavior of limit-cycle oscillators," Phys. Rev. Lett. 65, 1701-1704.

Meucci, R., Salvadori, F., Ivachenko, M. V., Naimee, K. A., Zhou, C., Arecchi, F. T., Boccaletti, S. \& Kurths, J. [2006] "Synchronization of spontaneous bursting in $\mathrm{CO}_{2}$ lasers," Phys. Rev. E 74, 066207.

Mirollo, R. E. \& Strogatz, S. H. [1990] "Amplitude death in an array of limit-cycle oscillators," J. Statist. Phys. 60, 245-262.

Mokhov, I. I., Smirnov, D. A., Nakonechny, P. I., Kozlenko, S. S., Seleznev, E. P. \& Kurths, J. [2011]
"Alternating mutual influence of El Nino/Southern Oscillation and Indian monsoon," Geophys. Rev. Lett. 38, L00F04.

Nakagawa, N. \& Kuramoto, Y. [1993] "Collective chaos in a population of globally coupled oscillators," Progr. Theoret. Phys. 89, 313-323.

Nakao, H. \& Mikhailov, A. S. [2009] "Diffusion-induced instability and chaos in random oscillator networks," Phys. Rev. E 79, 036214-1-5.

Neda, Z., Ravasz, E., Brechet, Y., Vicsek, T. \& Barabasi, A.-L. [2000] "The sound of many hands clapping," Nature 403, 849-850.

Pantaleone, J. [2002] "Synchronization of metronomes," Amer. J. Phys. 70, 992-1000.

Pecora, L. M. \& Carroll, T. L. [1990] "Synchronization in chaotic systems," Phys. Rev. Lett. 64, 821-824.

Pecora, L. M. \& Carroll, T. L. [1998] "Master stability functions for synchronized coupled systems," Phys. Rev. Lett. 80, 2109-2112.

Pikovsky, A., Rosenblum, M. \& Kurths, J. [2003] Synchronization: A Universal Concept in Nonlinear Sciences (Cambridge University Press, Cambridge).

Strogatz, S. [2003] SYNC: The Emerging Science of Spontaneous Order (Hyperion, NY).

Strogatz, S. H. [2000] "From Kuramoto to Crawford: Exploring the onset of synchronization in populations of coupled oscillators," Physica D 143, 1-20.

Sun, J., Bollt, E. M. \& Nishikawa, T. [2009] "Master stability functions for coupled nearly identical dynamical systems," Europhys. Lett. 85, 60011-1-5.

Uchiyama, S. [2012] "Flow version of statistical neurodynamics for oscillator neural networks," Physica A 391, 2807-2817.

Van Leeuwen, P., Geue, D., Thiel, M., Cysarz, D., Lange, S., Romano, M. C., Wessel, N., Kurths, J. \& Groenemeyer, D. H. [2009] "Influence of paced maternal breathing on fetalmaternal heart rate coordination," Proc. Natl. Acad. Sci. USA 106, 13661-13666.

Varela, F., Lachaux, J. P., Rodriguez, E. \& Martinerie, J. [2001] "The brainweb: Phase synchronization and large-scale integration," Nature Rev. Neurosci. 2, 229-239.

Wiesenfeld, K., Colet, P. \& Strogatz, S. [1996] "Synchronization transitions in a disordered Josephson series array," Phys. Rev. Lett. 76, 404-407.

Wu, C. W. \& Chua, L. O. [1995] "Synchronization in an array of linearly coupled dynamical systems," IEEE Trans. Circuits Syst.-I 42, 430-447.

Zhao, J., Hill, D. J. \& Liu, T. [2011] "Synchronization of dynamical networks with nonidentical nodes: Criteria and control," IEEE Trans. Circuits Syst.-I 58, 584594. 